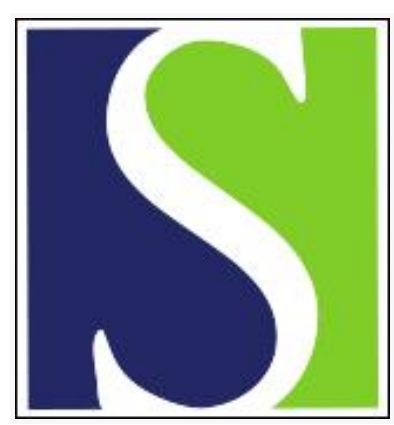

Scand J Work Environ Health 1991;17(5):302-311

https://doi.org/10.5271/sjweh.1698

Issue date: Oct 1991

A classification system for causes of occupational accidents for use in preventive strategies.

by Feyer AM, Williamson AM

Affiliation: National Institute of Occupational Health and Safety, Sydney, Australia.

This article in PubMed: www.ncbi.nlm.nih.gov/pubmed/1947915

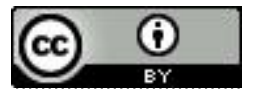




\title{
A classification system for causes of occupational accidents for use in preventive strategies
}

\author{
by Anne-Marie Feyer, PhD, Ann M Williamson, $\mathrm{PhD}^{1}$
}

\begin{abstract}
FEYER A-M, WILLIAMSON AM. A classification system for causes of occupational accidents for use in preventive strategies. Scand J Work Environ Health 1991;17:302-11. A comprehensive classification system, which allowed operational analysis of the events preceding accidents, was applied to the analysis of information surrounding the occurrence of all traumatic work-related fatalities in Australia in 1982-1984. The coded information included factors immediately antecedent to the accident leading to the fatality and factors removed in time which contributed to the occurrence of the accident. The complex network of events leading up to the accident, their interrelationships, and their relative contribution to causing the accident were examined. The results provide information about the use of accident analysis for the formulation of preventive strategies.
\end{abstract}

Key terms: accident analysis, prevention, work-related accidents.

Epidemiologic descriptions of the occurrence of occupational accidents provide information about the nature, distribution, and rate of work-related injury and death (1). They describe how, when, and where accidents occur and are therefore important starting points for identifying preventive strategies. However, classifying accidents by type or severity of injury, or by agent or place of injury, provides limited information about why accidents occur. Comprehensive knowledge about the causes of occupational accidents has the potential to provide much more specific information on which to base preventive efforts.

Several frameworks have been proposed for analyzing the causes of accidents (2-9). However, most of these frameworks have not been comprehensive in that they have not considered the range of possible causal factors and their interrelationships. The range of causal factors has been restricted both in the extent to which different types of factors are considered and also in the extent to which the wider circumstances surrounding the occurrence of the accident are included. In some instances, only one type of causal event, for example, human error $(2,7)$, or a broad general category of causal factor, for example, behavioral factors (3), has been considered in detail.

Alternatively, where a wide range of factor types has been included, it has been at a relatively superficial level. Consideration of some of the more complex causal factors, such as human error, has typically been much less detailed $(5,10)$.

Similarly, lack of comprehensiveness and detail has also been evident in the extent to which circumstances

\footnotetext{
1 National Institute of Occupational Health and Safety, Sydney, Australia.
}

Reprint requests to: Dr A-M Feyer, National Institute of Occupational Health and Safety, GPO Box 58, Sydney NSW 2001 Australia. surrounding the accident are considered. Although several frameworks have considered only those events immediately precipitating the accident $(8,11)$, more typically, most accident analysis frameworks recognize the potential importance of both immediate precipitating causes and the wider circumstances surrounding the occurrence of the accident $(5,12-15)$. However, analysis of the wider circumstances has often not been as detailed as for events occurring immediately before the accident $(12,13)$.

The nature of the relationships between factors is another important dimension of the analysis of accident causes. Several studies have considered the relative sequential nature of causal factors such that they define when different factors occur in the accident sequence $(5,9,12,13)$. The relative causal nature of relationships between different factors in the accident sequence has not been studied. Since it is clear that all factors are not of equal causal significance, having information about the relative importance of factors in accident causation must improve the specificity with which targets for prevention can be defined (14). This information, however, is rarely gathered.

Some models of accident causation have attempted to acknowledge the causal contribution of factors. Frameworks based on fault-tree analysis have provided the most restrictive view of the causal contribution of factors $(6,9,12,16)$. They have sought to identify the necessary and sufficient relationships between the events in the accident sequence by use of logic rules. Other approaches have taken a more conceptual view of the relative importance of causal factors $(5,14)$, the events immediately precipitating the accident being seen as only symptomatic of the underlying or preexisting conditions which contribute to the occurrence of the accident. The underlying factors, located in the wider circumstances of the occurrence of the accident, 
are seen as causally more significant. The most parsimonious approach, adopted only rarely, has been to impose a weighting system in order to establish the relative importance of factors without any preconceptions about the role that either immediate or more removed factors might play (10).

Classification systems have been developed which recognize the need for the most comprehensive treatment possible of information available about the occurrence of the accident $(14,16)$. These systems have still to be applied to large banks of accident data. In part, the lack of application reflects the fact that the frameworks involve distinctions which can be specified at a theoretical level, but are often difficult to operationalize, especially when the process is retrospective. The specification of complex logical relations between events $(6,16)$, for example, is limited in applicability in retrospective accident analysis.

The aim of the present research was to design a comprehensive classification system for occupational accidents which would describe the array of causal factors, map their relative sequential relationships, evaluate the relative importance of factors in accident causation, and, yet, be operationally applicable. For the evaluation of its applicability, the classification system was applied to a large heterogeneous data base of occupational accidents.

\section{Methods}

The study population was derived from all workrelated traumatic deaths occurring throughout Australia in the years 1982-1984. The data were collected from coroners' reports in a study of work-related fatalities conducted by the National Institute of Occupational Health and Safety (as described in reference 1). Of the 1738 fatalities judged to be work-related by Harrison et al (1), 1020 were included in the current study. Cases were excluded on the following grounds: (i) case was not in active work at the time of the fatality, (ii) case was not of working age, that is, below 15 years or above 65 years of age, (iii) death occurred during work commuting, (iv) death due to a road traffic accident when the vehicle was not the person's normal workplace, and (v) death occurred when person was on recess from active work or was a bystander to work. Of the remaining cases, 130 were excluded because they did not contain enough information to be coded. The study therefore included all fatalities that occurred while the person was actively involved in work over the three-year period of study.

The coroners' reports were relatively comprehensive. They contained a textual account of the circumstances of the accident and subsequent fatality, as well as demographic information about the person, medical information regarding the nature of the injuries and cause of death, and details of all investigations into the causes of death.

\section{The classification system}

The classification system was developed to establish, from the information in the coroners' reports, the sequence of events surrounding and leading to the fatality. It was designed to allow coding of a sequence of up to three events which immediately preceded the accident and which led to the fatality, as well as any further factor(s), classified into one of eight possible categories, that made a direct contribution to the occurrence of the accident or fatality. These were titled precursor events (abbreviated to PE1, PE2, or PE3, depending on location in the sequence with respect to the accident) and contributing factors, respectively. Not all of the accidents involved the full range of precursor events and/or contributing factors. For the purposes of coding, time before the fatality referred to the relative sequential order of the events leading up to the fatality. Conventional units of time were therefore variable. In other words, PE1 was closest to the accident and PE2 occurred earlier than PE1 in the accident sequence, but the time separating the events could have varied from seconds to minutes to hours or even perhaps days. Figure 1 is a schematic representation of the possible event sequence.

The nature of each of the three possible precursor events was coded into one of the following categories: (i) environmental - events resulting from the location of the accident and are events that could not have been changed at that point in time, (ii) equipment - events resulting from breakage or malfunctions that occur in machinery or tools at that point in time, (iii) medical - events resulting from the person's current state of physical well-being, and (iv) behavioral - events resulting directly from human involvement. Each category was coded in terms of the certainty of evidence for its existence, with $1=$ yes, certainly; 2 =yes, probably; $3=$ yes, possibly; $4=$ indeterminate; $5=$ no.

Behavioral events were coded further in terms of who performed the behavior (the deceased or another person) and whether the behavior constituted an error (ie, the incorrect performance of standard operating procedure).

Errors were coded with the use of two well-known error classification systems to enable the assessment of the ease with which they can be used for the kind of data available in this study and their utility for preventive strategy formulation (17). They were Swain's (18) task-related error classification and Rasmussen's (7) classification of the functional level of behavior during which the error occurred. The results of this coding have been discussed elsewhere (17).

The nature of the contributing factors was coded into the following categories: (i) environment - factors resulting from the location of the accident occurring at an earlier time, (ii) equipment - factors associated with the design of machinery, tools, personal protective equipment or safety equipment, (iii) work practice - factors involving poor or risky standard operating procedures accepted by management and/or 


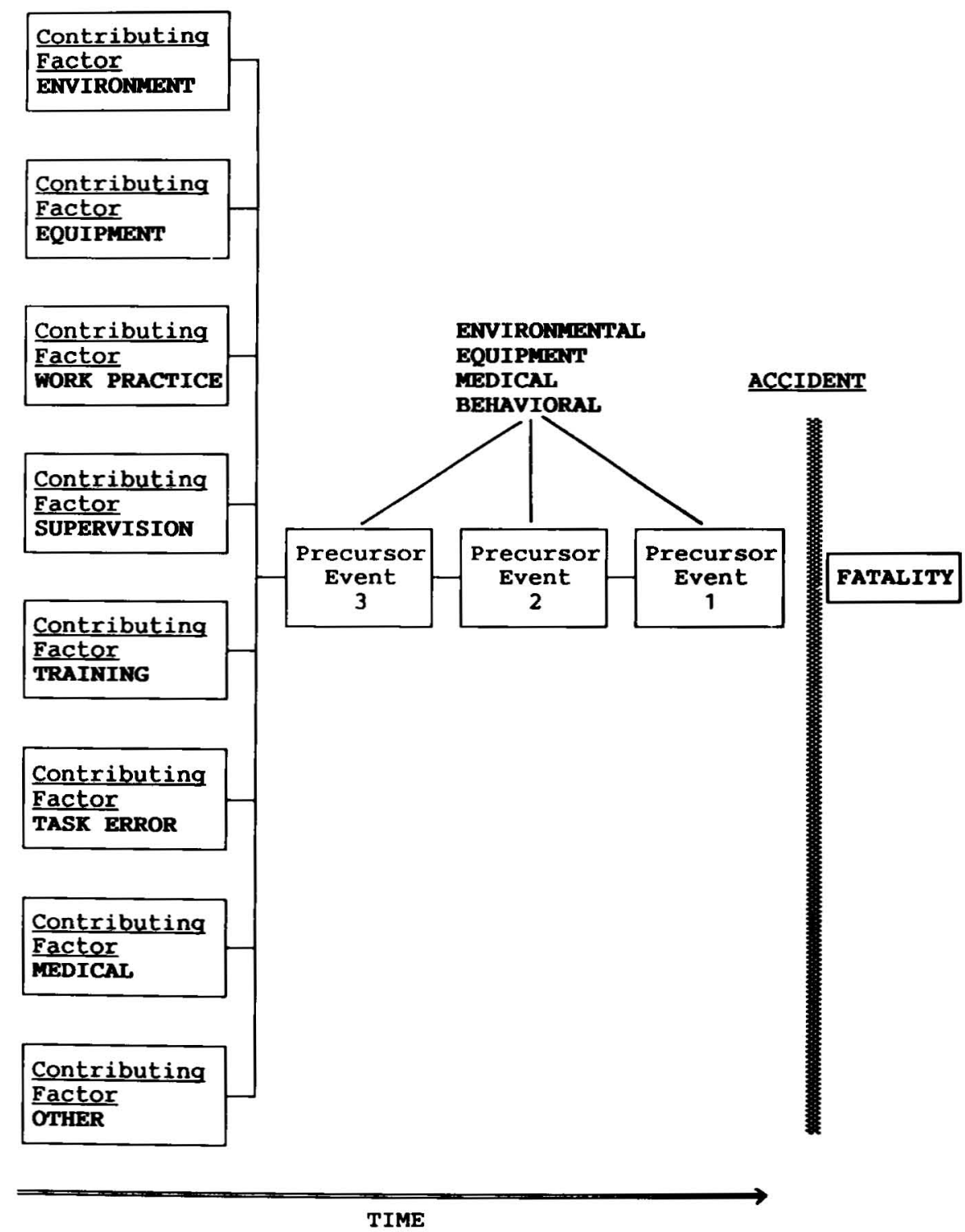

Figure 1. Schematic representation of the event sequence used for coding accidents.

personnel (this included separate categories of poor upkeep or misuse of equipment, (iv) supervision - factors relating to inadequate charge of workers, (v) training - factors relating to inadequate training of workers, (vi) task error - factors relating to incorrect performance of duty, (vii) medical - factors involving physical well-being at an earlier time, and (viii) other - factors such as alcohol/drug involvement, delays in receiving medical treatment and social aspects. The coding for contributing factors was again in terms of the certainty of evidence for the existence of the contributing factor.

One factor that was not included in the present classification system was injuring energy. All injury is most directly caused by energy release in excess of injury thresholds (19). Thus energy transfer was assumed to occur in all cases in the present study, and therefore the classification of its presence or absence would have been redundant. Classification of the nature of the energy involved is highly relevant to the nature, agent, mechanism, and severity of injury. In contrast, the focus of the present classification system was on the causes of the accident leading to injury. Therefore, it was those factors that initiated and influenced the build-up of energy which were coded.

Information was also collected on demographic details, details of the person's job at the time of death, the nature of their usual job (if different), the use of personal protective or other safety equipment, and the degree of forewarning of the accident. 


\section{Ranking of causal factors}

The precursor events and contributing factors leading up to the fatality were ranked in terms of their significance for the occurrence of the fatality. The chain (or chains) of events and factors were ranked such that the root cause was deemed as the most significant (scored " 1 "), and occurrences following directly from the root were ranked by diminishing degrees as significance decreased (ie, 2, 3, 4, etc). The root causes were termed prime causes. An important feature of the ranking procedure was that the prime cause or causes of an accident could be located in the contributing factor categories or the precursor event sequence. Therefore, it was possible for contributing factors to be ranked as more causally influential than precursor events in any given accident. This feature of the ranking procedure allowed the separation of causal importance and temporal importance of events; events were coded both in terms of proximity to the accident and causal significance such that the two were not necessarily equivalent. It was also possible to code more than one prime cause for any accident sequence.

The coding instrument was developed by three coders test-coding a random sample of 30 cases. The results were then discussed and the coding instrument modified to balance the scope of information collected with the quality of information available. Once the instrument was finalized, a single coder coded all cases.

\section{An example}

The following is a hypothetical example of the coding of an accident scenario into event categories and the ranking of those event categories for causal significance.

Scenario. While standing on a catwalk above large machinery, a worker leaned too closely into the path of the operating machinery. He was dragged into the machinery. The guard usually on the catwalk had been removed several days previously to facilitate repair of the machinery and had not been replaced. The general work area was poorly lit.

Coding. Precursor event sequence:

PE1 (error: leaned into path of machinery; RANK: 3)

PE2 (none)

PE3 (none)

Contributing factors:

Environment (poor lighting; RANK: 2)

Work practice (railings removed; RANK: 1)

Interpretation. The event immediately preceding the accident was an error on the part of the worker when he leaned too far into the path of the machinery. Operating the process without the usual safety railing allowed the error to occur, while the poor lighting in the work area made its occurrence much more likely.

\section{Reliability}

Inter- and intrarater reliability of two aspects of coding were evaluated. First, the reliability with which the same event category sequences were coded to describe the cases was evaluated. Two independent coders coded 40 randomly selected cases, and their frequency of agreement with the main coder was calculated. The main coder recoded these 40 cases after a period of six months had elapsed, and the frequency of agreement between the two coding occasions was calculated.

The second aspect of coding that was evaluated was the reliability with which the relative causal importance of the events could be ranked. There were two parts to evaluating the reliability of assigning rank to events. First, ranks were assigned to the events coded in the 40 randomly chosen cases that have already been described. In other words, where differences occurred in categorizing cases into event sequences, the coders had ranked different event category sequences for the same case. Since the event category sequences into which cases are coded critically influences the importance assigned to an event in the sequence, this was not an unambiguous evaluation of the reliability of ranking per se. Therefore, the following additional method of evaluating ranking reliability was used: 40 randomly selected cases, coded into event category sequences by the main coder, were ranked by the two independent coders. In this procedure each coder ranked the same event category sequences. This procedure allowed an evaluation of the reliability of the ranking of events in a sequence in relation to each other, independently of coding the events into categories.

To measure the association between pairs of coders for the ranking of the events, the Guttman coefficient of monotonicity was used. A monotone trend of $Y$ on $\mathrm{X}$ implies that, as $\mathrm{X}$ increases, any change in $\mathrm{Y}$ is onedirectional with the possibility that $Y$ can occasionally remain constant (20). The coefficient varies between 0 and 1.

\section{Results}

\section{Reliability}

When 40 randomly selected cases were coded by two independent coders, the percentage of agreement with the main coder for coding the cases into event category sequences was $84 \%$ for one coder and $82 \%$ for the other coder. The percentage of agreement when the main coder recoded these 40 cases after a period of six months had elapsed was $89 \%$.

When complete cases were both coded and ranked independently, the Guttman coefficient was calculated 
on an event-by-event basis for all possible events in the sequence (ie, for the three precursor events and the eight contributing factors) because of the possibility of different numbers of events in the sequence for different coders. The mean Guttman coefficient for one independent coder compared with the main coder was 0.68 (SD 0.25). For the other independent coder, the mean Guttman coefficient was 0.83 (SD 0.13). The Guttman coefficient for the main coder compared on the two coding occasions separated by six months was 0.89 (SD 0.09).

When coded cases were ranked, that is, the same sequences of event categories were ranked, by the two independent coders and these were compared with the rankings assigned by the main coder, the Guttman coefficient was $\mathbf{0 . 9 9}$ for both independent coders.

\section{Summary of involvement of event categories}

Figure 2 shows the frequency of involvement of the major event categories and the percentage of cases in which each category was ranked as a prime causal factor. Errors, poor work practices, and environmental factors were the factors most frequently involved in accident causation. Errors showed the highest frequency of involvement, occurring in more than two-thirds of the cases. Environmental factors, either as a percursor event or as a contributing factor, were the next most common and were present in $62.4 \%$ of the cases.
Poor work practices were involved in almost half of all the cases. The remaining categories each occurred in less than $15 \%$ of the cases.

Human error at some point in the sequence was also the most common prime causal factor. In fact, almost half of all the cases involved error as a prime cause. Poor work practices were involved as a prime cause of the fatality in approximately one-third of the total number of cases. Although environmental factors were among the most frequently involved factors, they were not commonly involved as prime causal factors.

\section{Patterns of prime causal rankings}

Examination of the distribution of prime causal rankings in the event sequences revealed that prime causal factors were at least as prominent in the contributing factors as they were in the precursor sequence. In over half of all the cases $(55.6 \%)$ there was one prime causal factor in the precursor event sequence. Similarly, in $56.2 \%$ of the cases, there was at least one prime causal factor in a contributing factor category. Furthermore, in a little over one-third of the cases $(37 \%)$, prime causal factors were located exclusively among the contributing factors. In $43.8 \%$ of the cases, prime causal factors were located exclusively in the precursor event sequence.

Multiple prime causes occurred the most commonly as a combination of precursor events and contrib-

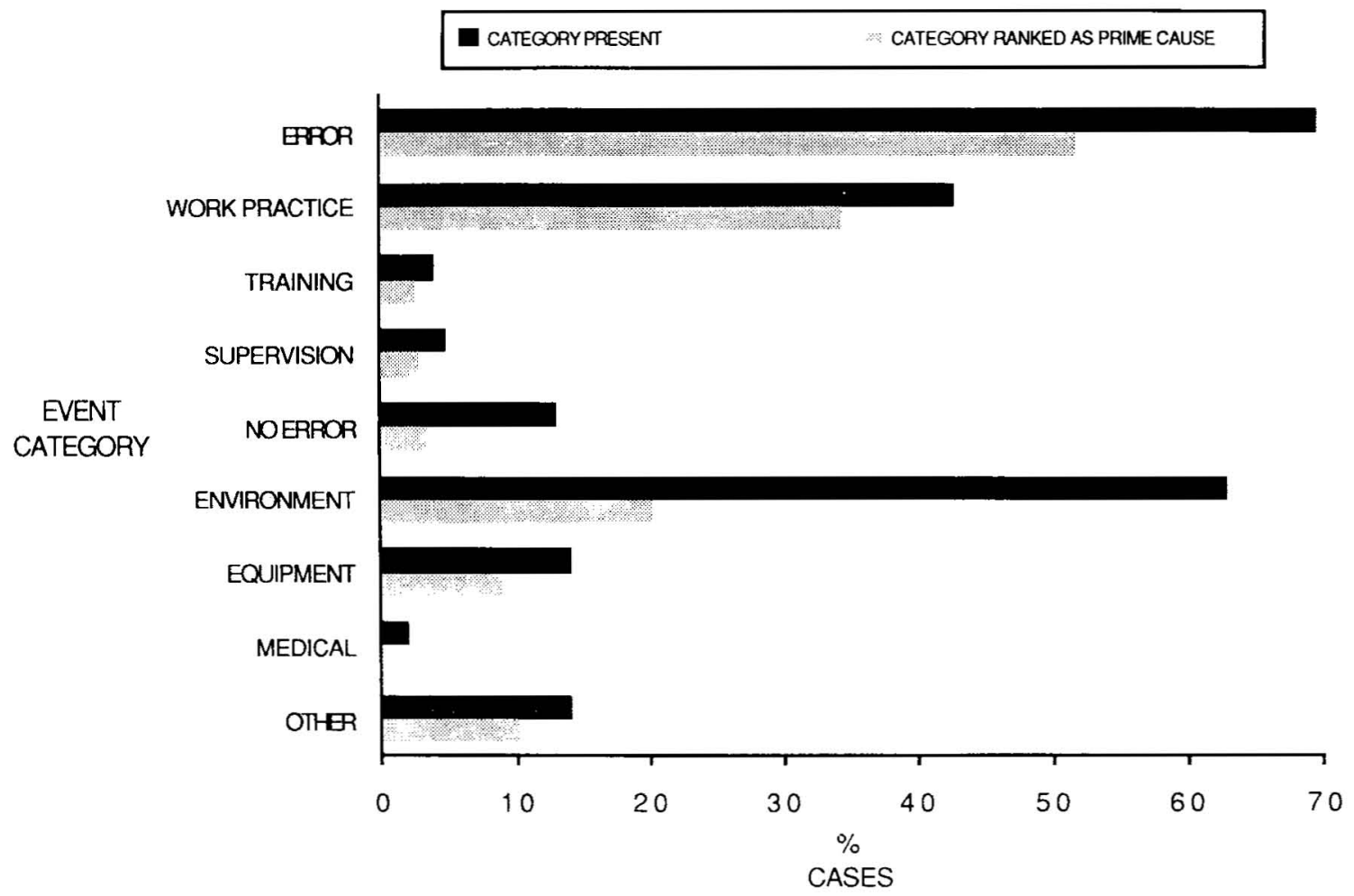

Figure 2. Major categories in the event sequences preceding occupational fatalities, showing percentages of cases with each type of event at least once in the accident sequence and the percentage of cases in which each was ranked as a prime cause of the accident. 
uting factors. In $23.2 \%$ of the cases there was at least one contributing factor with a ranking of " 1 " in addition to an event in the precursor sequence with a ranking of " 1 ." In contrast, multiple prime causes were located in the precursor sequence only in $7.4 \%$ of the cases; they were present in the contributing factors only in $10 \%$ of the cases.

\section{Patterns of event sequences and rankings}

The precursor event sequences were examined for consistent patterns. Twelve patterns were found, each representing at least $2 \%$ (about 20 cases) of the cases. Four of these patterns accounted for $66 \%$ of all cases in the sample. These four major patterns are discussed in the following text.

Two of these event sequences involved a behavioral event at PE1. (See figure 3.) For ease of display, patterns involving other event categories at PE1 were terminated at PE1 and nonmajor patterns involving a behavioral event at PE1 were terminated at PE2 or PE3 in figure 3. The number in parentheses under each PE label in the figure refers to the percentage of total cases represented by the sequence to that point.

Together, the two main patterns accounted for $34.5 \%$ of all the cases, while the remaining patterns with a behavioral event at PE1 accounted for only $4.1 \%$ of the cases. In the more common of the two patterns ( $25.7 \%$ of all cases), the behavioral event at PE1 was the sole event in the precursor event sequence. In the other pattern, accounting for $8.8 \%$ of the cases, the behavioral event at PE1 was preceded by another behavioral event at PE2. An important distinguishing feature of these two precursor sequences relates to the relative causal importance of the behavioral event at PE1. Specifically, when the pattern consisted of a sole behavioral precursor event, that event was ranked as a prime causal factor in the majority of cases $(66.3 \%$ ) represented by the sequence. However, when the pattern was one in which the behavioral event at PE1 was preceded by another behavioral event at PE2, it was the earlier event (at PE2) which was ranked as a prime causal factor for the majority of cases $(57.3 \%$ ) represented by the sequence, rather than the event at PE1 (27\% of cases).

Figure 3 also shows the contributing factors associated with each precursor sequence. The number in parentheses under each contributing factor label refers to the percentage of cases represented by the particular sequence which also showed involvement of that contributing factor. The number not in parentheses refers to the percentage of cases represented by the precursor sequence for which the contributing factor was ranked as a prime cause.

Both patterns showed almost identical involvement of contributing factors. The work practice category was involved in about one-third of the cases, the environment category and the other category both being represented in approximately $20 \%$ of the cases. With respect to ranking, both the work practice category and the other category were ranked as prime causal factors in more than $70 \%$ of the cases in which they were present, while the environment category was ranked as a prime causal factor much less frequently (in $22 \%$ of the cases for one pattern and in $0 \%$ of the cases for the other).

The remaining two major patterns were ones in which an environmental event immediately preceded the accident. (See figure 4.) Together, the two patterns accounted for about one-third of all the cases (32.1\%), in approximately equal proportions.

In one of the patterns, the environmental event at PE1 was the sole event in the precursor event sequence. In the other pattern, the environmental event at PE1 was preceded by a behavioral event at PE2. The relative causal importance of the environmental event at PE1 was changed dramatically with the presence of a preceding behavioral event. When the environmental event was the sole event in the precursor sequence, it was ranked as a prime causal factor in $41.4 \%$ of the cases represented by the sequence. In contrast, when the pattern was one in which it was preceded by a behavioral event at PE2, the environmental event at PE1 was ranked as a prime causal factor in only $17.6 \%$ of the cases represented by the sequence, while the earlier behavioral event was ranked as a prime causal factor in the majority of cases $(65.5 \%)$.

The work practice, other, and environment categories were again the main contributing factors involved. Work practice was the most common, and it was ranked the most often as a prime causal factor. Environmental factors were again less causally influential than contributing factors from either the work practice or the other category.

For the pattern in which an environmental event immediately before the accident was the only precursor event, there was a substantially increased involvement of behavioral contributing factors, work practice and task errors at an earlier time being the contributing factors occurring the most often. Furthermore, these contributing factors were predominantly ranked as prime causal factors.

\section{Discussion}

Inter- and intrarater reliability was high both for coding cases into event category sequences and for assigning rankings of relative causal importance to the event categories. This finding suggests that the classification system is viable for describing the network of events preceding accidents and for evaluating their relative causal importance. The system therefore provides an important starting point for the development of a data-driven base for the formulation of preventive strategies.

Human error, poor work practices, and environmental factors were found to be the most frequent antece- 
dents of the fatalities. However, the prime causes of accidents were not necessarily those factors which were the most frequently involved. Error at some point in the sequence was the most frequently involved factor. It was also the most common prime causal factor. In contrast, environmental factors, which were involved at some point almost as frequently as error, were prime causal factors in only a minority of cases. In other words, for most cases involving environmental factors, other factors in the accident sequence were of greater causal significance.

Nor were the prime causes of the accidents necessarily those factors which occurred the most closely in time to the accident. In one-third of the cases there were no prime causal factors located in the precursor event sequence. That is, the root cause of the accident

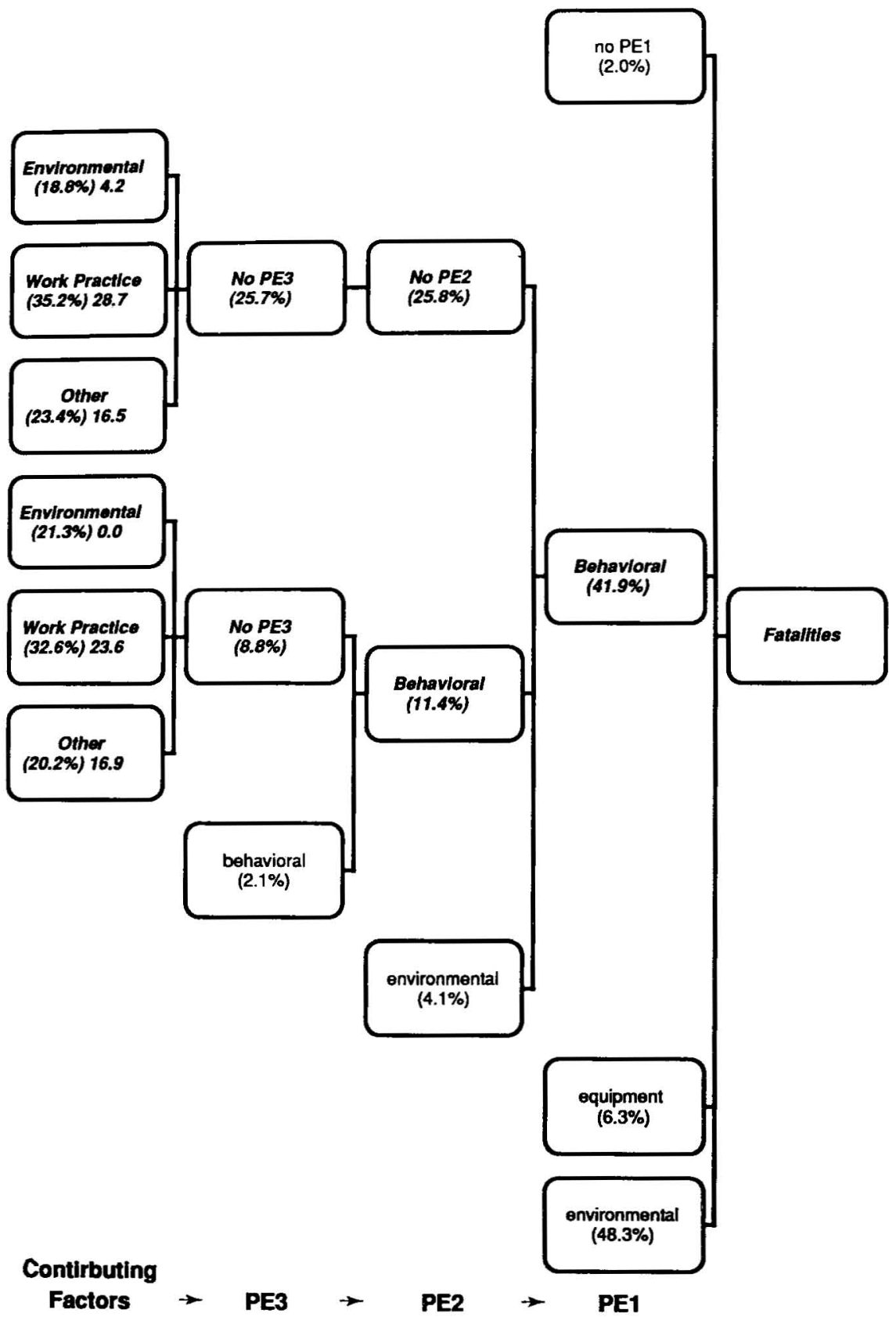

Figure 3. Major patterns of event sequences with a behavioral event at precursor event 1 (PE1). The number in parentheses under each PE refers to the percentage of total cases represented by the sequence to that point. For the contributing factors, the number in parentheses refers to the percentage of cases represented by the PE sequence which also showed involvement of that contributing factor, and the number that is not in parentheses refers to the percentage of cases represented by the sequence for which the contributing factor was ranked as a prime cause. 


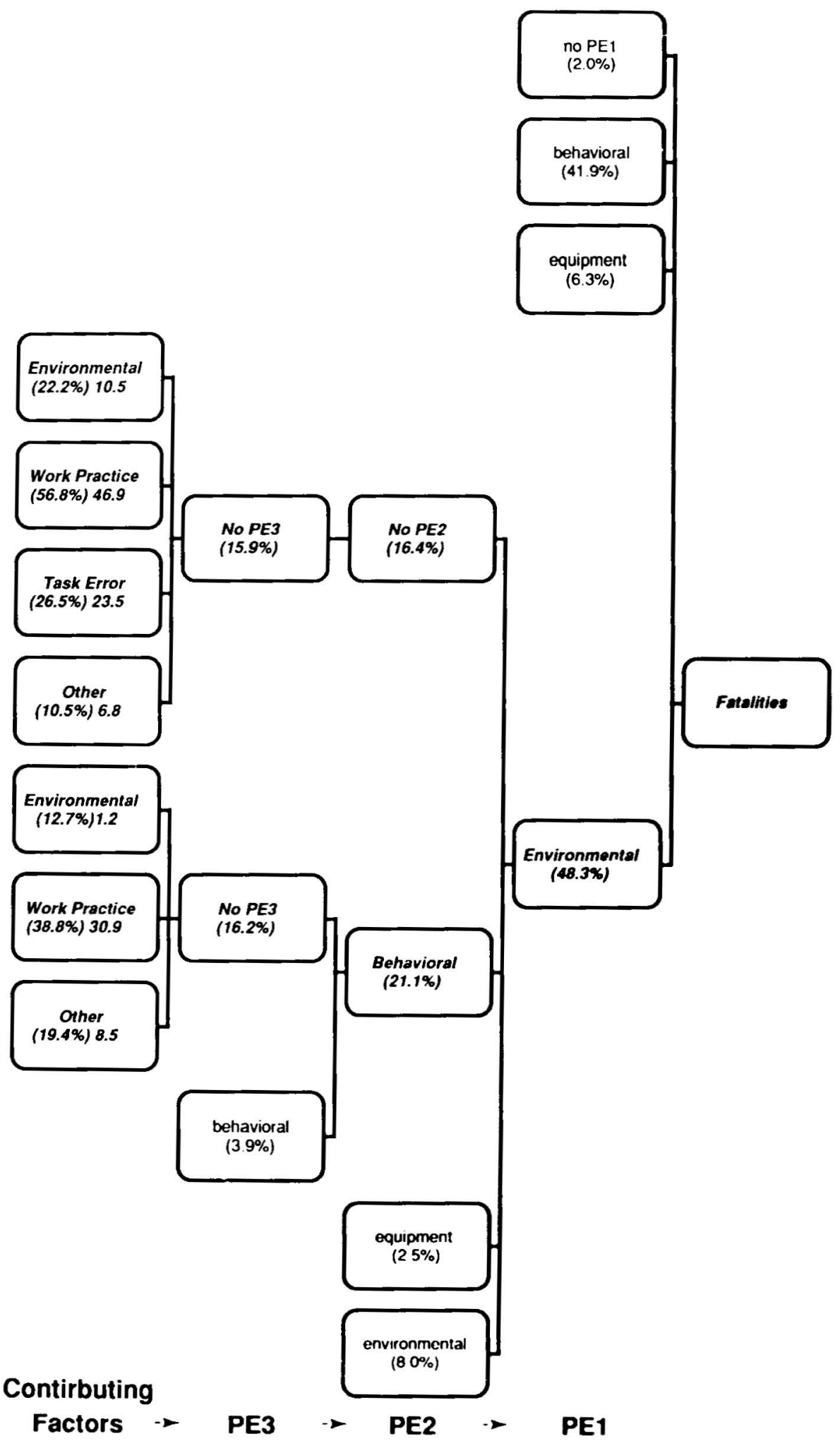

Figure 4. Major patterns of event sequences with an environmental event at precursor event 1 (PE1). The number in parentheses under each PE refers to the percentage of total cases represented by the sequence to that point. For the contributing factors, the number in parentheses refers to the percentage of cases represented by the PE sequence which also showed in volvement of that contributing factor, and the number that is not in parentheses refers to the percentage of cases represented by the sequence for which the contributing factor was ranked as a prime cause. 
for these cases was located outside the event sequence leading directly to the accident.

Not only did prime causal factors occur at various locations in the accident sequence, but multiple prime causes were also relatively frequent. In about one-third of the cases, at least two prime causal factors occurred in the accident sequence, most commonly as a combination of precursor events and contributing factors.

Knowing where in the accident causation sequence a particular factor is likely to occur and how important it is likely to be provides a useful starting point for identifying targets for prevention. However, because the accidents were shown to be multicausal, more information is needed about the causal pathways whereby factors exert their influence. Without this information it is very difficult to identify where in the accident sequence, and with what sort of strategy, it may be possible to intervene the most effectively.

A striking characteristic of these results was that there were relatively few patterns of causal pathways. Indeed, the majority of the accidents were accounted for by one of four major precursor event sequences consisting of exclusively behavioral events, exclusively environmental events, or a combination of these two categories. Furthermore, similar networks of contributing factors were found to be associated with each of the four main sequences, specifically the environment, the work practice, and the other categories. The main component of the other category was found to be drug and alcohol involvement.

Factors occurring early in the sequence were identified as much more influential in causing the fatalities than events closer to the fatality. When two events occurred in the event sequence, the earlier event (PE2) was generally ranked as more important. This earlier event was a behavioral one for all patterns; therefore it was not possible to determine whether its influence was due to the nature of the event or its location in the sequence. The fact that behavioral contributing factors were always ranked highest regardless of the rest of the accident sequence suggests that a combination of these characteristics (ie, location and nature of the event) is important.

These results clearly indicate that the nature and influence of events occurring earlier in the sequence has important implications for drawing inferences about preventive strategies. Being able to identify that preexisting poor work practices and earlier task errors, and to a less extent drug and alcohol involvement, were frequently the predisposing inputs for the events which immediately precipitated the accident provides highly specific targets for prevention.

The need for the application of comprehensive accident analysis systems to large banks of accident data has often been highlighted $(3,14,21)$. The present study constitutes the first attempt to do so. The classification system was designed to provide the sort of information that can be used to identify targets for prevention. It comprehensively and reliably describes the antecedents of the accidents, their interrelationships, and their relative causal importance. The results confirmed that accidents are the outcome of a complex network of interrelated factors which are not equivalent in causal significance. The findings also demonstrated that when information is available about the nature, temporal location with respect to the accident, and causal role of all of the factors in the antecedent network, then the targets for prevention can be much more specifically defined.

The level of analysis reported in this presentation has provided only an overview of the major causal networks preceding this large group of fatalities. It provides some insights into the contributors to workrelated deaths in Australia. A more-detailed analysis of the involvement of human error has already been completed (17). Further analysis will focus on the differences in accident causation across industry, the detailed investigation of the nature and role of poor work practices, and the role of risk taking in accident causation.

\section{Acknowledgments}

We are very grateful to Ms D Worthington for her work in coding the fatalities and to Dr D Cairns for his invaluable advice regarding the presentation of the data. We would like to thank Dr M Frommer and Mr $\mathrm{J}$ Harrison for allowing access to data of their study on work-related fatalities.

The views expressed in this article are those of the authors and do not necessarily reflect those of the $\mathrm{Na}$ tional Occupational Health and Safety Commission.

\section{References}

1. Harrison JE, Frommer MS, Ruck EA, Blyth FM. Deaths as a result of work-related injury in Australia, 19821984. Med J Aust 1989;150:118-25.

2. Corlett EN, Gilbank G. A systematic technique for accident analysis. J Occup Accid 1978;2:25-38.

3. Hale AR, Glendon AI. Individual behaviour in the control of danger. Amsterdam: Elsevier, 1987.

4. Johnson WG. MORT safety assurance systems. New York, NY: Marcel Dekker, 1980.

5. Kjellen U, Larsson TJ. Investigating accidents and reducing risks - a dynamic approach. J Occup Accid 1981;3:129-40.

6. Leplat J. Accident analyses and work analyses. J Occup Accid 1978;1:331-40.

7. Rasmussen J. Human errors: a taxonomy for describing human malfunction in industrial installations. J Occup Accid 1982;4:311-33.

8. Shannon HS, Manning DP. The use of a model to record and store data on industrial accidents resulting in injury. J Occup Accid 1980;3:57-65.

9. Wagenaar WA, Groeneweg J. Accidents at sea: multiple causes and impossible consequences. Int J Man-Mach Stud 1987;27:587-98.

10. Shaw BE, Sanders MS. Research to determine the frequency and cause of injury accidents in underground mines. In: Proceedings from the Human Factors Society 31st annual meeting, Oct 19-23. Santa Monica, CA: 
Human Factors Society, 1987:926-30.

11. Ryan JP. A study of safety in man-machine systems. In: Asfour SS, ed. Trends in ergonomics/human factors IV. Amsterdam: Elsevier, 1987:505-11.

12. Leplat J. Accidents and incidents production: methods of analysis. J Occup Accid 1982;4:299-310.

13. Tuominen R, Saari J. A model for analysis of accidents and its application. J Occup Accid 1982;4:263-73.

14. Wagenaar WA, Reason J. Types and tokens in road accident causation. Ergonomics 1990;33(10/11):1365-76.

15. Glendon AI, Hale AR. Accidents to young people on the UK Youth Opportunities Programme. In: Debus G, Schroiff $\mathrm{H}-\mathrm{W}$, ed. The psychology of work and organization. Amsterdam: Elsevier, 1986:329-36.

16. Johnson WG. MORT: the management oversight and risk tree. J Saf Sci 1975;7(1):4-15.

17. Williamson A, Feyer AM. Behavioural epidemiology as a tool for accident research. J Occup Accid 1990; 12
$(1-3): 207-22$.

18. Swain AD. A method for performing a human factors reliability analysis. Albuquerque, NM: Schandia National Laboratories, 1963. (Monograph SCR-685).

19. Haddon W. The changing approach to the epidemiology, prevention and amelioration of trauma: the transition to approaches etiologically rather than descriptively based. Am J Public Health 1968;58:1431-8.

20. du Toit SHC, Steyn AGW, Stumpf RH. Graphical exploratory data analysis. New York, NY: Springer Verlag, 1986.

21. Hale $\mathbf{A R}$, Hale M. A review of the industrial accident research literature. London: Her Majesty's Stationery Office, 1972.

Received for publication: 30 July 1990 